\title{
Multi-station LiDAR scanning-based hierarchical features for generation of an allometric stem volume model
}

\author{
Yuan Sun $\odot,{ }^{\mathrm{a}, \mathrm{b}, *, \dagger}$ Xiuyun Lin,,${ }^{\mathrm{a}, \mathrm{b}, \dagger}$ Yulin Gong, ${ }^{\mathrm{a}}$ Jiawen Jiang, ${ }^{\mathrm{a}}$ \\ Yanli Zhang, ${ }^{c}$ and Xiaorong Wen ${ }^{\mathrm{a}, \mathrm{b}}$ \\ ${ }^{a}$ Nanjing Forestry University, Co-Innovation Center for Sustainable Forestry in Southern China, \\ Nanjing, China \\ ${ }^{b}$ Nanjing Forestry University, College of Forestry, Nanjing, China \\ ${ }^{c}$ Stephen F. Austin State University, Arthur Temple College of Forestry and Agriculture, \\ Nacogdoches, Texas, United States
}

\begin{abstract}
Terrestrial laser scanning technology has developed rapidly, and substantial data have been accumulated in dynamic forest monitoring. Point cloud data of standing trees not only provide tree parameters but also show three-dimensional tree structure. Selection of the key parameters from the point cloud data is a prerequisite for volume estimation of standing trees. Our study collected three phases of data over 5 years from Liriodendron chinense plantation forest. A series of the height-related characteristic parameters were extracted from the scanned points of each tree stems, including a proposed new parameter and the height cumulative percentage $\left(H_{z} \%\right)$. The upper diameter accuracy obtained by multi-station scanning is high, and the correlation coefficient with manually measured data is 0.9864 . The shape of the upper tree trunk extracted by the point cloud is equivalent to that of the sample trees (height of 10 to $20 \mathrm{~m}$ ) with points at $H_{25 \%}$ and $H_{50 \%}$ of the height. These two parameters also show a high correlation with volume. Results show that $H_{25 \%}$ can better associated with tree volume, with $R^{2}$ at 0.951 , 0.957 , and 0.901 at three stages, respectively. The volume dynamic change calculated by model 2 is linearly correlated with the rate in point cloud restoration, the intercept is -0.081 , and the slope is 1.14. Compared with previous researches, the volume model established based on point cloud hierarchical parameters in this study could be used for monitoring the dynamic volume changes in Liriodendron forest. The $H_{z}$ value extracted from multi-station scanning point cloud data could be used to represent the dynamic forest structure. The results of this study contribute to further development of terrestrial laser scanning-based modeling and estimation methods for individual tree and forest growth, thereby improving the accuracy of forest inventories estimation and providing better tools for decision-making processes. () The Authors. Published by SPIE under a Creative Commons Attribution 4.0 Unported License. Distribution or reproduction of this work in whole or in part requires full attribution of the original publication, including its DOI. [DOI: 10.1117/1.JRS.15 .028503]
\end{abstract}

Keywords: height accumulative percentage; dynamic change; terrestrial laser scan; tree volume; allometric model; Liriodendron chinense.

Paper 200751 received Oct. 22, 2020; accepted for publication May 18, 2021; published online Jun. 3, 2021.

\section{Introduction}

Long-term dynamic monitoring and evaluation of forest growth and development constitute the main objectives and research directions of forest management. ${ }^{1}$ The application of three-dimensional (3D) laser point cloud data has facilitated various landscapes and plot-level applications ranging from the structure and distribution of forest to the dynamic changes of forest resources. ${ }^{2-7}$

Tree growth can be determined through changes in the most typical tree attributes such as diameter at breast height (DBH) and height $(h)$. Traditional measurements of standing

\footnotetext{
*Address all correspondence to Yuan Sun, yuan.sun@njfu.edu.cn

${ }^{\dagger}$ These authors contributed equally to this work.
} 
parameters and volume modeling require data from destructive logging to have accurate values. ${ }^{3,6}$ Some investigations performed in the early 2000 s explored the capability of terrestrial laser scanning (TLS) technology to measure forestland factors. ${ }^{4,6,8}$ It can accurately measure the structural properties of live stumpage, such as the stem curves, which are difficult to be directly measured by conventional methods. ${ }^{3,9}$ However, preliminary research focuses on precision of point cloud, ${ }^{2}$ modeling method, ${ }^{4,10}$ and selection of appropriate parameters. ${ }^{5,11}$ Some research showed result that current diameter-based allometric models for forest biomass are highly uncertain. ${ }^{12}$ And some researchers found that the point cloud-derived diameters in a taper curve matching approach performed the best at estimating diameters at the lowest parts of the stem $\left(<30 \%\right.$ of the total tree height). ${ }^{13}$

In recent years, many researches focused on the application of multi-phase scanning data. Several studies concentrate on measuring single-tree attributes by means of TLS other than airborne LiDAR system because airborne laser is blocked by canopy, the identification accuracy of understory trunk morphology, especially ground diameter and DBH, is low. Kaasalainen et al. ${ }^{14}$ obtained the multi-temporal point cloud information of loblolly pine with TLS. The point cloud characteristic parameters of each time phase were extracted and the correlation between them and the dynamic estimation model of AGB was established based on the characteristic parameters of different phases. Kaasalainen et al. ${ }^{14}$ were able to discover growth and changes in the structure of trees with repeated scans during a 3-year period. Mengesha et al. ${ }^{15}$ reported that multi-temporal TLS data enabled the accurate estimation of stem volume growth. Luoma et al. ${ }^{7}$ obtained the laser-point cloud data of four sample plots in Nucsio National Park in Finland in 2008 and 2017 and extracted tree measurement factors such as DBH, tree height, shape number, and shape ratio from the laser-point cloud. It described the change of a single tree in 9 years with average relative change of trunk volume is $65.0 \%$. So far, most structural measures are focused on or limited to individual parameter.

TLS has shown considerable promise in obtaining highly accurate estimates of the tree diameter and stem curve, and the point cloud density can accurately reflect the spatial distribution of tree trunks from top to bottom. ${ }^{16}$ However, several studies have reported underestimates for TLS-based tree height. ${ }^{6}$ In terms of the data size and geometric complexity, the segmentation of characteristic TLS parameters has great advantages. ${ }^{1}$ Models can be established to extract the structural parameters of stumpage using point cloud coordinate information. ${ }^{17}$ A multi-station scanning method was used in the study. After splicing, denoising, and normalization, the point cloud data have a relatively uniform point distribution regardless of the type of equipment. The next phase is to obtain complete information about the stumpage diameter, tree height, and vertical distribution of the tree crown. An algorithm to extract a new hierarchical characteristic of TLS point clouds was developed. Stem analysis data were used to analyze the hierarchical structure parameters. Then the final models corresponding to the measured stumpage characteristics were developed to estimate volume change based on the characteristic parameter using data in three periods to provide a reference for applying TLS in monitoring tree volume and the dynamic forest changes.

\section{Materials and Methods}

\subsection{Study Site and Data Collection}

The study site was located within the Experimental Forest of Nanjing Forestry University to the west of Wuqi Hill in Jiangsu Province, China. The annual average temperature is $15.5^{\circ} \mathrm{C}$ with a maximum of $29^{\circ} \mathrm{C}$ in summer and a minimum of $2^{\circ} \mathrm{C}$ in winter. The average elevation and slope are $\sim 150 \mathrm{~m}$ and $20 \mathrm{deg}$, respectively. The yellow brownish soil is $\sim 60$-cm-thick and has a gravel content of $20 \%$ and surface humus content of $1.5 \%$. An even-aged forest of Chinese tulip trees (Liriodendron chinense) was planted with a spacing of $3 \mathrm{~m} \times 3 \mathrm{~m}$ in March 1981, and the sample plot has a size of $40 \mathrm{~m} \times 25 \mathrm{~m}$.

The point cloud data of the sample plot were collected in three phases in winter in January 2014, 2018, and 2019, when the trees were leafless. The plantation had very little undergrowth and therefore is appropriate for research using TLS. A Leica Scan Station C10 was used in 2014, 


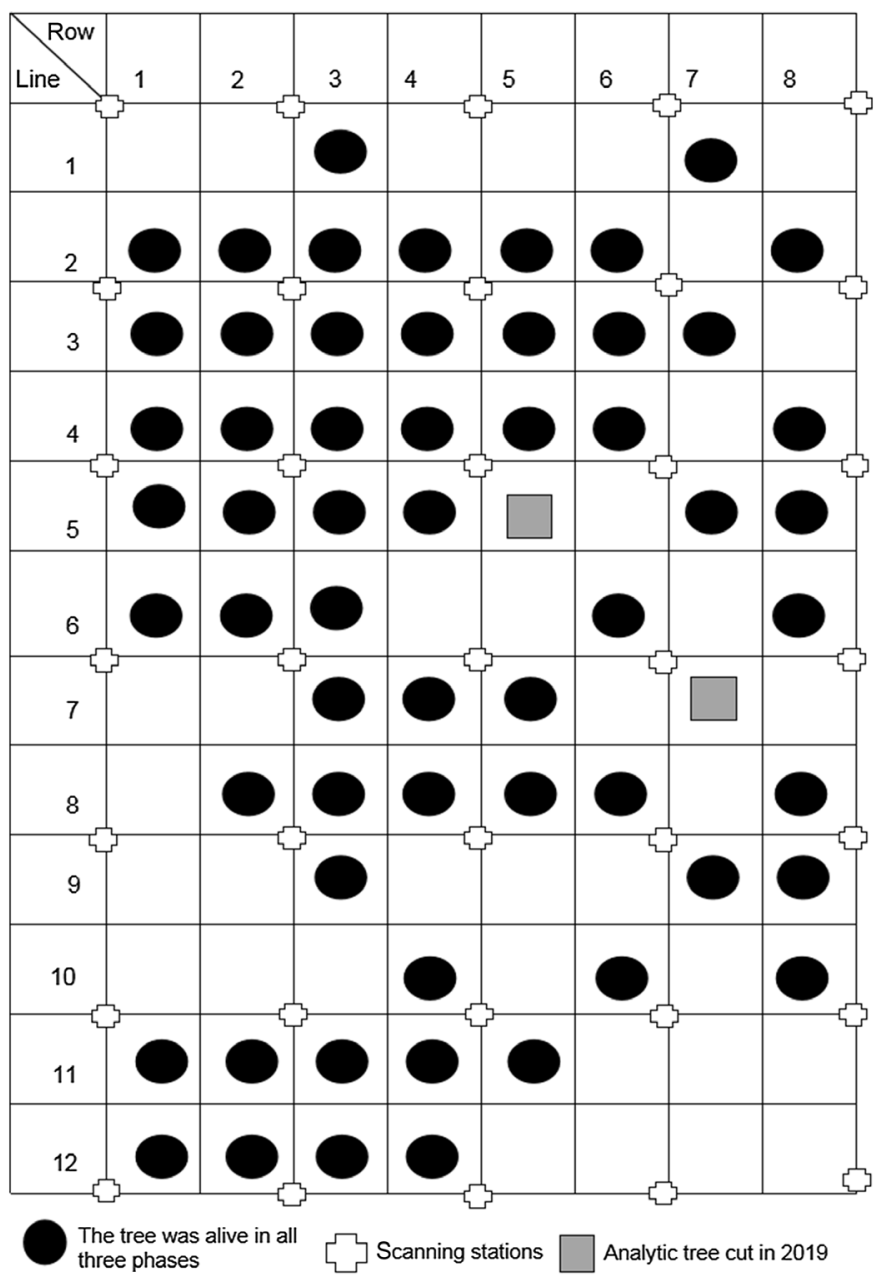

Fig. 1 Sketch of the tree distribution in the study area.

where the scanning angle was set to 270 deg vertically and 360 deg horizontally, and the scanning rate was 50,000 dots/s. The upper and lower targets were used to facilitate the first data registration. In 2018 and 2019, a Riegl VZ-400i laser scanner with an original digital echo, realtime waveform processing, and multi-beam transceiver processing technologies was used, target was not needed, and it is capable of accepting infinite echoes with scanning angles of $100 \mathrm{deg}$ vertically and 360 deg horizontally and has a scanning rate of 500,000 dots/s.

At the same time, sample data were obtained by conducting a manual inventory check of each standing tree. In the inventory, the tree height was measured by a Leica D810 altimeter, and the $\mathrm{DBH}$ and ground diameter were both measured with a diameter tape. Two trees were selected according to mean DBH from the sample data in 2019: the fifth tree in row 5 (DBH of $29.2 \mathrm{~cm}$ ) and the seventh tree in row 7 (DBH of $33.1 \mathrm{~cm}$ ). Figure 1 shows a schematic of the distribution of Chinese tulip trees at the sample site in 2014, 2018, and 2019, and the gray squares represent the locations of the trees utilized for stem analysis in 2019.

\subsection{Method}

The sample plot was scanned at multiple stations, and all data were merged together through registration and splicing. Then all points were imported into the same $3 \mathrm{D}$ coordination system. ${ }^{18}$ The registration precision was $0.0045 \mathrm{~m}$ in 2014 and $0.0038 \mathrm{~m}$ in 2018 and 2019. LiDAR360 v2.2 was used to extract the digital elevation model (DEM) and carry out the normalization procedure. Because the trees were leafless, the degree of overlap between individual tree point clouds obtained at the canopy level was almost negligible. Consequently, the crown of each 
individual tree could be clearly identified from the top view of the sample plot data. To fully obtain information regarding the 3D forest, this study employed manual identification and subsetting to position and segment each tree to facilitate the subsequent extraction of the standing timber parameters.

\subsubsection{Extracted tree height}

To extract the tree height of each tree obtained by manual recognition and subsetting, the height of a single tree was calculated by the point cloud height difference, which is the difference in the peak cloud height of a single tree minus the height of the ground point cloud as shown in the following equation:

$$
H=Z_{\max }-Z_{\min }
$$

\subsubsection{Point cloud hierarchical characteristic analysis}

Although point cloud density features can accurately reflect the state of the target spatial distribution, most density features are based on plane density analysis, which cannot fully reflect the real density characteristics of 3D point clouds. From a hierarchical view of the point cloud of a standing tree, this research proposed the concept of the height cumulative percentage $\left(H_{z}\right)$ of the point cloud to study the association of $H_{z}$ with other important forest resource parameters, such as the stem volume.

$H_{z \%}$ represents the cumulative total height $(z \%)$ of all points in the cloud at a lower or equal height. Before calculating $H_{z}$, the point cloud of each individual tree should be normalized (with the DEM as the ground datum) and then sorted according to each point's height. MATLAB 2014a was used to process the data and to calculate $H_{z}$ based on Eqs. (2) and (3):

$$
\begin{gathered}
H_{z}=h_{m}, \\
z \%=\frac{\sum_{i=1}^{m}(h 1+h 2+h 3+h 4+\ldots+h i)}{\sum_{i=1}^{n}(h 1+h 2+h 3+h 4+\ldots+h i)} \times 100 \%,
\end{gathered}
$$

where $n$ is the total number of points for the point cloud of a tree and $h_{i}$ is the height of point $i$. $m$ denotes the number of points, in which Eq. (8) is balanced, and $h_{m}$ is assigned to $H_{z \%}$. Taking the tree in Fig. 2 as an example, the total number of data points in the point cloud is 189,648, and the summed height of all points is $2,269,368 \mathrm{~m}$. The height $h_{m}$ of point $m$ is $25.28 \mathrm{~m}$, which accounts for $75 \%$ of the total cumulative height; therefore, $H_{75}=h_{m}=25.28 \mathrm{~m}$.

Other hierarchical features of the point cloud, such as the mean height (Ht mean), standard deviation of the height $(\mathrm{Ht}$ std dev), variance of the point cloud height ( $\mathrm{Ht}$ var), quartile of the height (Ht IQ), average absolute deviation (AAD) of the height and median absolute deviation (MAD) from the median height, were also extracted as candidate variables for the subsequent stem volume regression (Table 1).

Among these characteristic variables, they are $\mathrm{AAD}=\frac{\sum_{i=1}^{n}\left(\left|h_{i}-\bar{h}\right|\right)}{N}, \mathrm{Ht}$ stddev $=$ $\sqrt{\frac{1}{N-1} \sum_{i=1}^{n}\left(h_{i}-\bar{h}\right)^{2}}$, and Ht Var $=\frac{\sum_{i=1}^{n}\left(h_{i}-\bar{h}\right)^{2}}{n}$ (where $n$ is the number of points in the point cloud of a tree, $h_{i}$ is the height of point $I$, and $\bar{h}$ is the mean height of all points).

\subsubsection{Extracted diameters at various heights}

Circle or cylinder fitting have most commonly been used for deriving DBH estimates from TLS. ${ }^{1}$ Based on the single tree point data, 3D point cloud data were converted into two-dimensional point cloud data; then the least squares method was used to fit each circle to extract the diameter at a specific height. Figures 3(a) and 3(b) show that the data pertaining to single trees were sliced. Excessively thin slices of point cloud data will result in insufficient data for calculating the diameter, whereas overly thick slices will reduce the extraction efficiency. ${ }^{9}$ This study controlled the slice thickness at $0.1 \mathrm{~m}$. Rings were sliced at intervals of $1 \mathrm{~m}$ from bottom through the trunk, 
Total points $(n): 189,648$

The total points' height: $2,269,368 \mathrm{~m}$

Cumulative height ratio is:

$$
75 \%=\frac{\sum_{i=1}^{m}(h 1+h 2+h 3+h 4+\cdots+h i)}{\sum_{i=1}^{n}(h 1+h 2+h 3+h 4+\cdots+h i)} \times 100 \%
$$

Where $h_{1}$ is the height of the $i-$ th point and $h_{m}$ is the height corresponding to the $m-$ th point

$$
H 75=h_{m}=25.28 m
$$
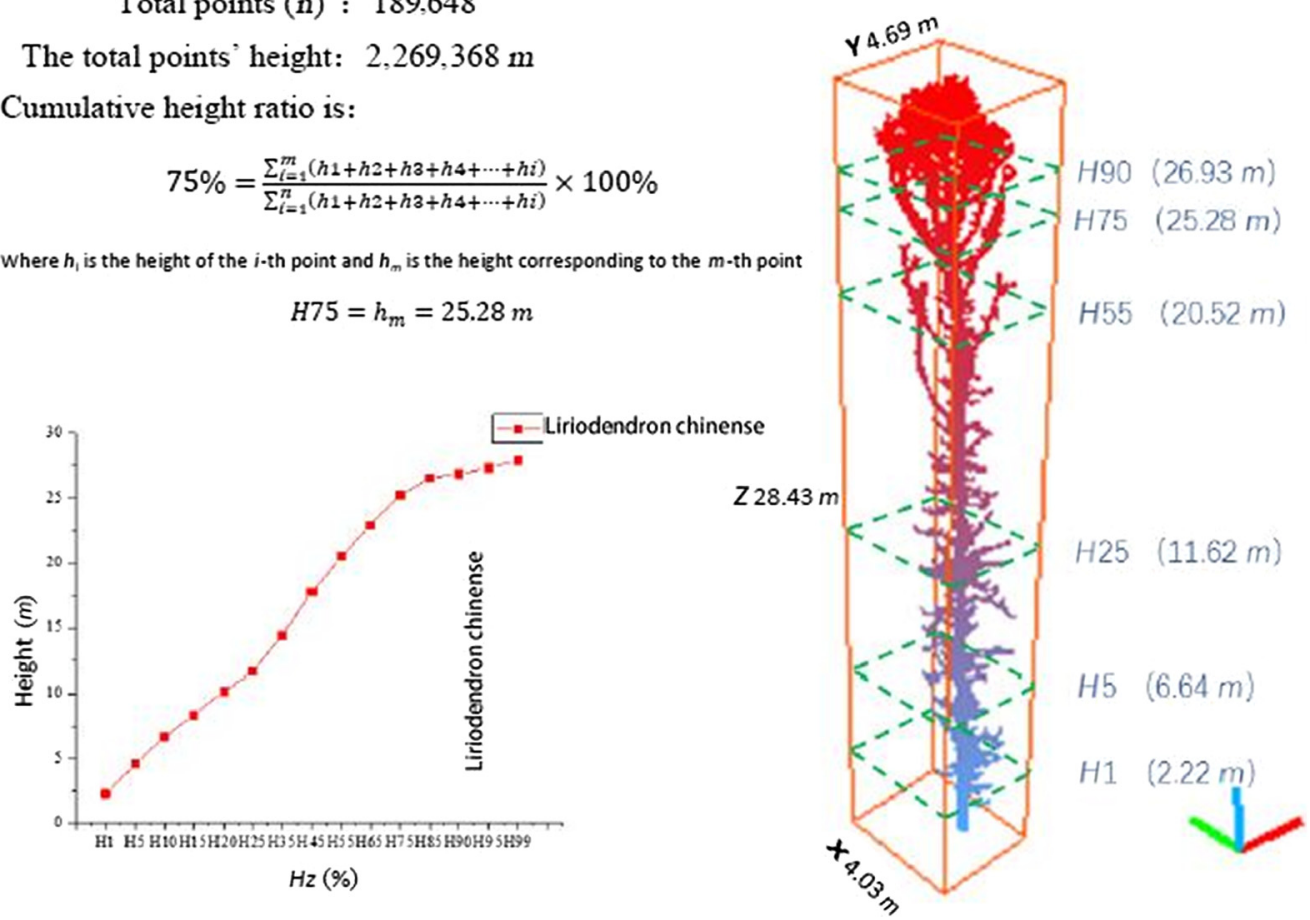

Fig. 2 Example of the height cumulative percentage $\left(H_{z \%}\right)$.

Table 1 Point cloud characteristic variables.

\begin{tabular}{ll}
\hline $\begin{array}{l}\text { Characteristic } \\
\text { variable }\end{array}$ & \multicolumn{1}{c}{ Description } \\
\hline $\mathrm{Ht}$ mean & Average height of the cloud point above the ground \\
$\mathrm{Ht}$ std dev & Standard deviation of the point cloud height \\
$\mathrm{Ht}$ var & Variance of the point cloud height \\
$\mathrm{Ht}$ IQ & Quartile distance of the point cloud height \\
$\mathrm{AAD}$ & Average absolute deviation \\
MAD median & Median absolute deviation from the overall median \\
\hline \hline
\end{tabular}

as shown in Fig. 3(b). Then the least square method was used to fit the diameter of the upper part of the stem. Detail method can be found in related research. ${ }^{1,9}$

\subsubsection{Obtaining the volume of each tree}

Through the method mentioned above, the research obtained three stages of point clouds of each tree along the tree height. The area segment quadrature method was used to calculate the standing timber volume of the central section of each tree. ${ }^{19}$ According to the previous step, the central diameter of each segment was extracted, using the cylinder volume formula to calculate volume. The remaining segment $(<2 \mathrm{~m})$ was treated as the tip of the tree, using the cone volume formula to calculate volume. The stem volume of each Chinese tulip tree (L. chinense) in the sample plot could be calculated with Eq. (6):

$$
V=l \sum_{i=1}^{n} g_{i}+\frac{1}{3} g^{\prime} l^{\prime},
$$




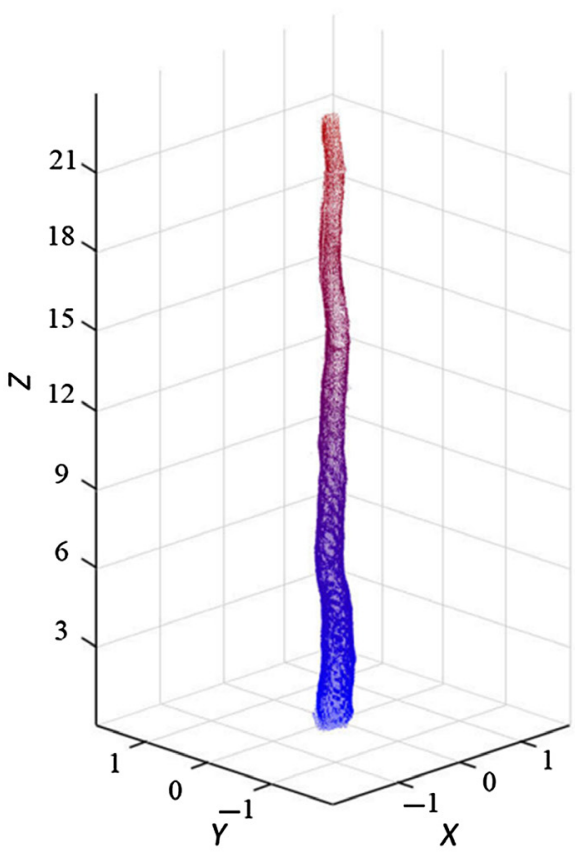

(a)

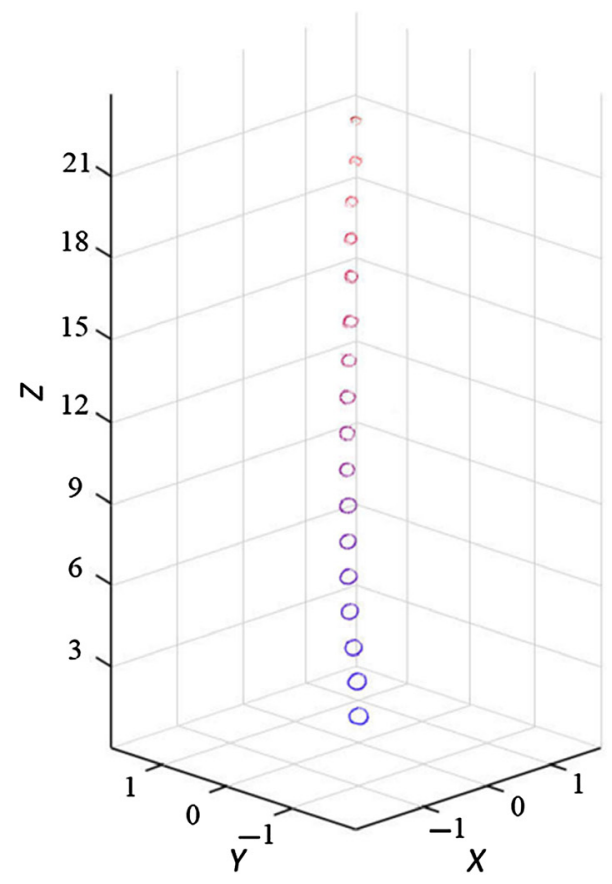

(b)

Fig 3 Extract upper diameter for a single tree: (a) tree trunk constituted by point cloud and (b) circle fitting for upper diameter.

where $V$ is the stem volume, $g_{i}$ is the cross-sectional area of each segment, $l$ is the length of the segment, $g^{\prime}$ is the cross-sectional area of the tip, $l^{\prime}$ is the length of the tip, and $n$ is the number of segments.

\subsection{Modeling and Verification}

To find the most suitable parameters for the volume model, the linear equation form shown in Eq. (5) was employed. According to the number of selected parameters, the formula was extended to obtain a unary primary volume model and a binary primary volume model ${ }^{20}$ Hence, three types of models were established. First, $H_{z}$ and DBH were taken as variables to conduct the modeling. Second, volume modeling was carried out by taking $H_{z}$ as the lone variable. Third, $H_{z \%}$ and other hierarchical features were modeled as variables:

$$
V=a_{0}+a_{1} x_{1}+a_{2} x_{2}+\cdots+a_{j} x_{j}, \quad j=1 \ldots m .
$$

Pearson's correlation coefficient $(P)$ was calculated between each extracted feature parameter and volume $(V)$. Feature parameters with a $P$ value larger than 0.6 were selected and used as candidate variables for the volume model. ${ }^{6}$ The parameters were calculated using the multivariate stepwise regression of SPSS19 software, where the significance level of the model parameters was set as 0.05 , and the equations with high collinearity were eliminated using the variance inflation factor (i.e., VIF > 10) variable using the VIF.

Then the binary volume equation ${ }^{19}$ was applied as a reference model [Eq. (6)] to establish the volume model based on the regression relationship between the DBH and tree height. This model reflects that certain stem characteristics have extensive regional applicability. DPS 17.1 software was used for the modeling and parameter estimation:

$$
V=a_{0}(D B H)^{a_{1}}(H)^{a_{2}}
$$

where $V$ is the volume value, $a_{i}$ denotes the coefficients, and $x_{i}$ represents the independent variables of the equation. 
To evaluate and verify the models, the coefficient of determination $\left(R^{2}\right)$, root-mean-square error (RMSE), and $F$ value were utilized as the evaluation indices for the model. The computation equations are as follows:

$$
\begin{gathered}
R^{2}=1-\frac{\sum_{i=1}^{n}\left(y_{i}-\hat{y}_{i}\right)^{2}}{\sum_{i=1}^{n}\left(y_{i}-\bar{y}_{i}\right)^{2}}, \quad i=1, \ldots, n, \\
\operatorname{RMSE}=\sqrt{\frac{1}{(n-1)} \sum_{i=1}^{n}\left(y_{i}-\hat{y}_{i}\right)^{2},} \\
F=\frac{\sum_{i=1}^{n}\left(\hat{y}_{i}-\bar{y}_{i}\right)^{2}}{m} / \frac{\sum_{i=1}^{n}\left(y_{i}-\hat{y}_{i}\right)^{2}}{n-m-1} .
\end{gathered}
$$

In the above equations, $y_{i}$ is the measured value, $\bar{y}_{i}$ is the measured mean value, $\hat{y}_{i}$ is the value predicted by the model, $n$ is the number of samples, and $m$ is the number of parameters in the equation.

The 10-fold cross-validation method was used to verify the prediction effect of the model. All individual tree data used for the modeling were randomly divided into $n$ subsamples under the condition that the prediction model parameters had already been determined. One subsample was randomly selected as verification data (to be used in the subsequent fitting model for validation), whereas the remaining $(n-1)$ samples were used for training. The results were verified with 10 iterations, each subsample was validated once, and the mean value of $n$ results was obtained as the estimated value of the model. ${ }^{20}$ The prediction accuracy of the model was verified by building a scatter plot between the estimated values of the model and the TLS-extracted values.

Dynamic changes in the volume of the Chinese tulip tree were analyzed based on the data from the three periods. The volume of stumpage in the three periods was acquired using the characteristic parameter volume prediction model, and the dynamic change in the volume was calculated according to the diameter classes. The changes were compared with the actual volume change computed by the point cloud, and the feasibility of applying the inverted volume prediction model to analyze the dynamic changes in timber volume was evaluated.

\section{Results and Analysis}

\subsection{Verification of the Extraction with Stem Analysis}

First, the extraction of the upper diameter should be confirmed. Data from the two sample trees are shown in Fig. 4 in a scatter plot of the upper diameters extracted from the point cloud and the corresponding diameters of the two trees. The extracted values have a high correlation $\left(R^{2}=0.9864\right)$ with the manually measured values and show no significant difference. Accordingly, the following features extracted from the point cloud can be researched.

Then data from the stem analysis were used to draw the taper curves as shown in Figs. 5(c) and 5(f). The tree trunk curves of the upper diameters provided by TLS point clouds are similar to those of the two sample trees [Figs. 5(b) and 5(e)]. Tree heights of 10 to $20 \mathrm{~m}$ represent the inflection points of the tree trunk. $H_{z}$ clearly increases with increasing height and reaches $\sim 10 \mathrm{~m}$ of the tree height at $H_{25 \%}$, which appears to be an inflection point; then $H_{z}$ reaches $\sim 20 \mathrm{~m}$ of the tree height at the value of $H_{50 \%}$ [Figs. 5(a) and 5(d)], which appears to be another inflection point and is followed by a gentle increasing trend for $H_{z \%}$.

\subsection{Features Extracted from the Point Cloud Data at Three Stages}

Following the methods above, this research extracted the tree structure parameters (DBH and tree height) and hierarchical features $\left(H_{z} \%\right.$ and Hmean) and counted the volume of each standing tree at all three stages. A summary of the stumpage features after normalization, denoising, extraction, and calculation is shown in Table 2. The average DBH of a single tree increased 


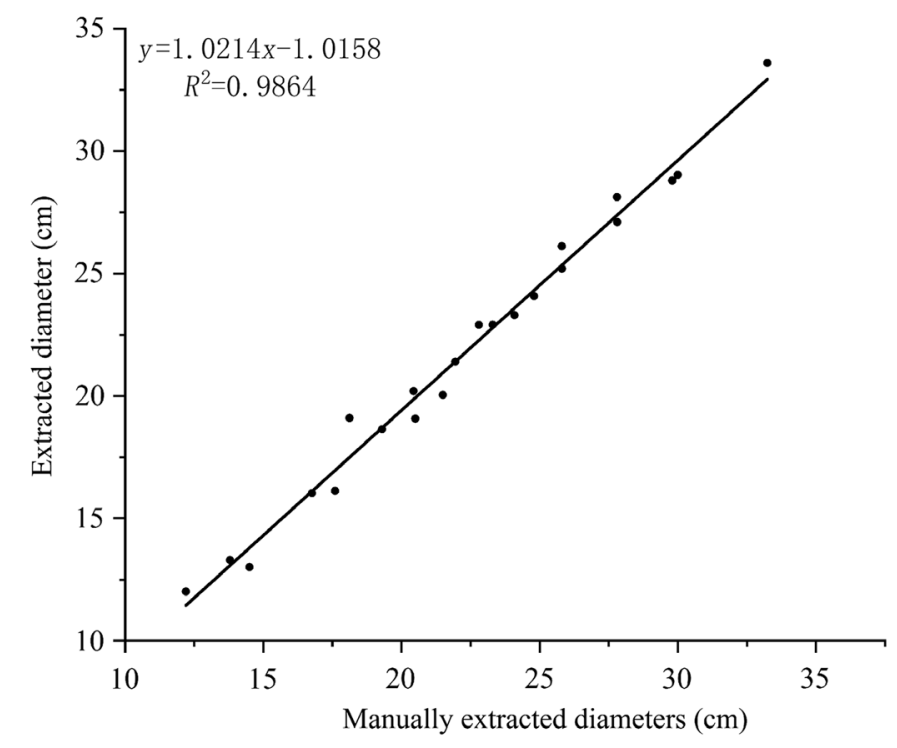

Fig. 4 Scatter plot of simple linear regression result for extracted diameters and manually measured diameters.

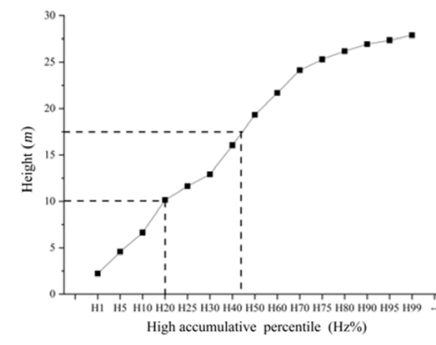

(a)

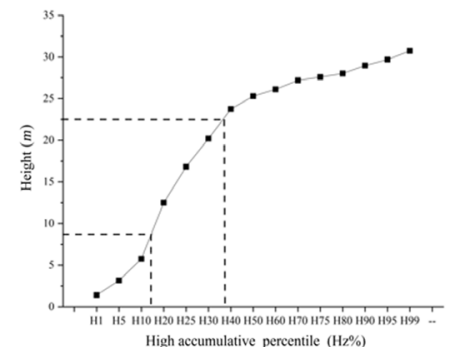

(d)

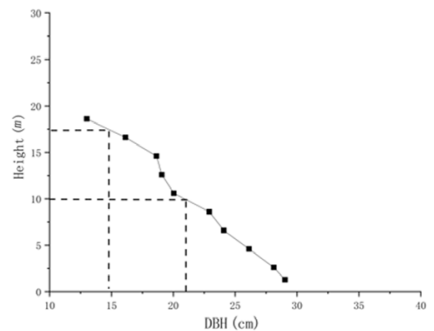

(b)

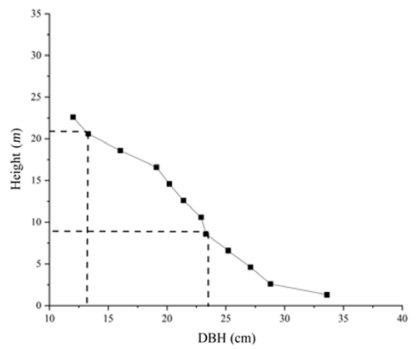

(e)

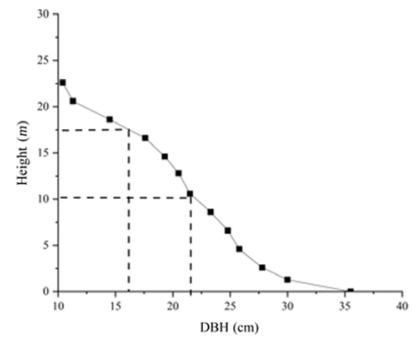

(c)

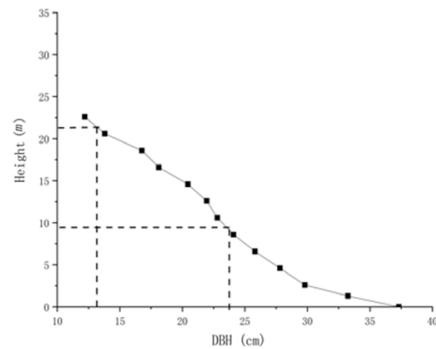

(f)

Fig. 5 Comparative analysis of high accumulative percentile and analytical wood stem curve: (a), (d) $H_{z}-H$; (b), (e) point cloud stem curve; and (c), (f) stem analysis curve.

significantly, and the average annual growth was stable at $0.3 \mathrm{~cm}$. The standard deviation of DBH growth was large: 5.95 in 2014, 6.49 in 2018, and 7.66 in 2019. The tree height and volume also increased annually. The average heights of $H_{75}, H_{55}$, and $H_{25}$, which refer to the spatial distribution of tree trunks from top to bottom, decreased annually in accordance with the law of tree growth.

\subsection{Volume Modeling Results}

A Pearson correlation analysis was conducted on individual trees, and the relationships between the tree height, $\mathrm{DBH}$, and hierarchical features with the tree volume determined by the point cloud data are shown in Table 3. According to the values from the point cloud data in the three 
Sun et al.: Multi-station LiDAR scanning-based hierarchical features for generation...

Table 2 Summary of features of a single tree in the three periods.

\begin{tabular}{|c|c|c|c|c|c|c|c|c|}
\hline Period & & $\begin{array}{l}\text { DBH } \\
(\mathrm{cm})\end{array}$ & $\begin{array}{c}\text { Tree } \\
\text { height }(m)\end{array}$ & $\begin{array}{c}\text { Volume } \\
\left(\mathrm{m}^{3}\right)\end{array}$ & $H_{75}$ & $H_{50}$ & $\mathrm{H}_{25}$ & Hmean \\
\hline \multirow[t]{3}{*}{2014} & Range & $\begin{array}{c}14.21 \text { to } \\
42.58\end{array}$ & $\begin{array}{c}17.11 \text { to } \\
32.40\end{array}$ & $\begin{array}{c}0.13 \text { to } \\
1.75\end{array}$ & $\begin{array}{c}11.98 \text { to } \\
29.72\end{array}$ & $\begin{array}{c}18.96 \text { to } \\
26.84\end{array}$ & $\begin{array}{c}14.18 \text { to } \\
20.13\end{array}$ & $\begin{array}{l}1.84 \text { to } \\
26.64\end{array}$ \\
\hline & Mean value & 25.96 & 28.30 & 0.69 & 24.51 & 20.51 & 11.44 & 15.65 \\
\hline & Standard deviation & 5.95 & 3.52 & 0.36 & 11.45 & 7.76 & 3.56 & 8.88 \\
\hline \multirow[t]{3}{*}{2018} & Range & $\begin{array}{c}15.21 \text { to } \\
45.04\end{array}$ & $\begin{array}{c}17.53 \text { to } \\
34.32\end{array}$ & $\begin{array}{c}0.14 \text { to } \\
1.96\end{array}$ & $\begin{array}{c}12.59 \text { to } \\
30.79\end{array}$ & $\begin{array}{c}7.75 \text { to } \\
27.99\end{array}$ & $\begin{array}{c}4.98 \text { to } \\
19.57\end{array}$ & $\begin{array}{l}4.09 \text { to } \\
28.70\end{array}$ \\
\hline & Mean value & 27.52 & 29.73 & 0.77 & 23.49 & 18.53 & 9.41 & 16.61 \\
\hline & Standard deviation & 6.49 & 3.93 & 0.40 & 12.1 & 7.51 & 2.93 & 9.24 \\
\hline \multirow[t]{3}{*}{2019} & Range & $\begin{array}{c}15.83 \text { to } \\
45.61\end{array}$ & $\begin{array}{c}17.67 \text { to } \\
35.00\end{array}$ & $\begin{array}{c}0.15 \text { to } \\
1.98\end{array}$ & $\begin{array}{l}9.22 \text { to } \\
22.02\end{array}$ & $\begin{array}{c}7.22 \text { to } \\
17.07\end{array}$ & $\begin{array}{c}2.92 \text { to } \\
8.96\end{array}$ & $\begin{array}{c}2.71 \text { to } \\
26.33\end{array}$ \\
\hline & Mean value & 27.87 & 30.12 & 0.80 & 15.29 & 10.47 & 5.55 & 10.93 \\
\hline & Standard deviation & 7.66 & 4.08 & 0.44 & 3.02 & 5.32 & 10.08 & 12.73 \\
\hline
\end{tabular}

Table 3 Correlation analysis of individual plant parameters and volume.

\begin{tabular}{|c|c|c|c|c|c|c|}
\hline & \multicolumn{2}{|c|}{2014} & \multicolumn{2}{|c|}{2018} & \multicolumn{2}{|c|}{2019} \\
\hline & Parameter & Correlation & Parameter & Correlation & Parameter & Correlation \\
\hline \multirow{2}{*}{$\begin{array}{l}\text { Structure } \\
\text { parameters }\end{array}$} & $\mathrm{DBH}$ & 0.9679 & DBH & 0.9679 & $\mathrm{DBH}$ & 0.9462 \\
\hline & Tree height & 0.6894 & Tree height & 0.4949 & Tree height & 0.7170 \\
\hline \multirow{10}{*}{$\begin{array}{l}\text { Hierarchical } \\
\text { features }\end{array}$} & $\mathrm{H}_{25}$ & 0.8662 & $\mathrm{H}_{25}$ & 0.7676 & $H_{80}$ & 0.7777 \\
\hline & $\mathrm{H}_{50}$ & 0.8424 & $H_{50}$ & 0.7654 & $H_{75}$ & 0.7618 \\
\hline & $\mathrm{Ht} I \mathrm{Q}$ & 0.8385 & $\mathrm{Ht} I \mathrm{Q}$ & 0.7561 & Ht stddev & 0.7170 \\
\hline & $A A D$ & 0.8363 & $A A D$ & 0.7449 & $\mathrm{H}_{50}$ & 0.6500 \\
\hline & $\mathrm{Ht}$ mean & 0.8300 & $\mathrm{Ht}$ mean & 0.7409 & $A A D$ & 0.6289 \\
\hline & $\mathrm{Ht}$ var & 0.8276 & $\mathrm{Ht}$ var & 0.7241 & Ht var & 0.5055 \\
\hline & Ht stddev & 0.8118 & Ht stddev & 0.6587 & MAD median & 0.4967 \\
\hline & $H_{75}$ & 0.7674 & $H_{75}$ & 0.6487 & $\mathrm{Ht}$ mean & 0.2973 \\
\hline & MAD median & 0.7561 & MAD median & 0.6360 & $\mathrm{Ht} I \mathrm{Q}$ & 0.2849 \\
\hline & $H_{80}$ & 0.7527 & $H_{80}$ & 0.5915 & $H_{25}$ & 0.1615 \\
\hline
\end{tabular}

periods, the DBH has the highest correlation with the tree volume, followed by the tree height, which conforms to the rule regarding the three elements of volume. Among the correlations between the hierarchical features and the tree volume, the highest correlation parameter in 2014 was $H_{25}$ and then $H_{50}$; the highest correlation parameter in 2018 was $H_{25}$ and then $H_{50}$; and the highest correlation parameter in 2019 was $H_{80}$ and then $H_{75}$. The various parameters involved in modeling the individual tree volume are listed in Table 4.

Thus the volume model was established using the hierarchical features, DBH and tree height as variables. The model results are shown in Table 4. Among them, the binary volume models 
Sun et al.: Multi-station LiDAR scanning-based hierarchical features for generation...

Table 4 Results of the volume model for individual trees.

\begin{tabular}{cccccc}
\hline \hline Time & Models no. & Parameter and coefficient & $R^{2}$ & $F$ value & RMSE $\left(\mathrm{m}^{3}\right)$ \\
\hline 2014 & $14-1$ & $v=0.106(\mathrm{DBH})^{2.035}(H)^{1.352}$ & 0.974 & 122.419 & 0.059 \\
$14-2$ & $v=-0.795+0.021\left(H_{25}\right)+4.847(\mathrm{DBH})$ & 0.951 & 570.604 & 0.083 \\
$14-3$ & $v=-0.617+0.073\left(H_{50}\right)-0.002\left(H_{80}\right)$ & 0.707 & 69.961 & 0.199 \\
$14-4$ & $v=-0.747+0.128\left(H_{75}\right)-0.062\left(H_{80}\right)$ & 0.612 & 45.690 & 0.229 \\
2018 & $18-1$ & $v=0.247(\mathrm{DBH})^{2.018}(H)^{1.075}$ & 0.974 & 140.053 & 0.066 \\
& $18-2$ & $v=-0.928+5.655\left(H_{25}\right)+0.016(\mathrm{DBH})$ & 0.957 & 661.538 & 0.086 \\
$18-3$ & $v=-0.699+0.051\left(H_{50}\right)+0.025\left(H_{80}\right)$ & 0.712 & 72.799 & 0.219 \\
$18-4$ & $v=-0.872+0.618\left(H_{75}\right)+0.009\left(H_{80}\right)$ & 0.642 & 52.983 & 0.244 \\
$19-1$ & $v=0.734(\mathrm{DBH})^{2.002}(H)^{0.734}$ & 0.919 & 288.254 & 0.039 \\
& $19-2$ & $v=0.062+0.092\left(H_{25}\right)+0.732(\mathrm{DBH})$ & 0.901 & 233.182 & 0.140 \\
$19-3$ & $v=0.494+0.011\left(H_{75}\right)+0.008\left(H_{85}\right)$ & 0.632 & 28.642 & 0.274 \\
\hline \hline & $19-4$ & $v=-1.147-0.034\left(H_{50}\right)+0.156\left(H_{80}\right)$ & 0.638 & 29.393 & 0.272 \\
\hline
\end{tabular}

(with $H$ and DBH as variables) are used as reference models and have the highest $R^{2}$ values $(0.974,0.974$, and 0.919 , respectively). After screening all the hierarchical features models, the three types of models with the best fitting effect are retained in Table 4, result shows that the models with hierarchical features $\left(H_{25}, H_{50}, H_{80}\right.$, and $\left.H_{75}\right)$ have better $R^{2}$ values $(0.951,0.957$, and 0.901). For predicting volume, model with $H_{25}$ is the best in this research. As shows in Fig. 5, that $H_{25}$ is the first inflection point along the trunk, revealing the efficacy of $H_{z \%}$.

Models 14-2, 18-2, and 19-2 are used to predict the individual tree volume, and scatter plots that show the correlations between the model-predicted values and TLS values of the single tree volume in all three periods are drawn [Figs. 6(a)-6(c)]. The plots reveal the precision of the models, which have relatively high $R^{2}$ values for 2019 (0.9603), 2018 (0.9501), and 2014 (0.949). All of the prediction lines are somewhat in accordance with the 1:1 verification line. Significant differences are not observed between the predicted and point cloud-extracted volumes. Therefore, the volume model with hierarchical parameters can be used as the volume model for each period.

Then the volume was counted for each DBH grade in each period, and the results are shown in Table 5. According to the previous steps, the volume of each tree was counted by the area segment quadrature method for the central section through the extracted point cloud data.

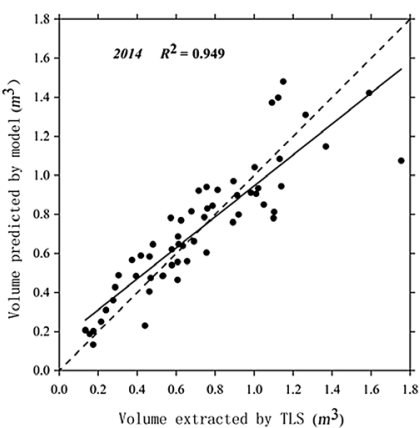

(a)

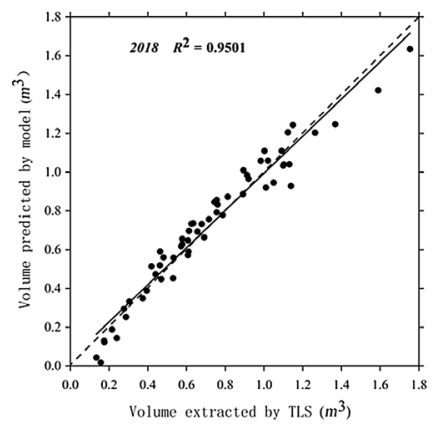

(b)

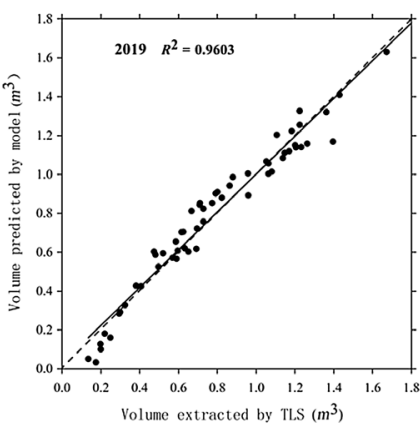

(c)

Fig. 6 Scatter plots of the correlation between the volume model-predicted volume and TLSextracted volume: (a) formula 2014-2, (b) formula 2018-2, and (c) formula 2019-2. 
Sun et al.: Multi-station LiDAR scanning-based hierarchical features for generation...

Table 5 Volume variations in DBH grades from 2014 to 2019.

\begin{tabular}{|c|c|c|c|c|c|c|c|c|c|c|}
\hline \multirow{2}{*}{$\begin{array}{l}\text { Diameter } \\
\text { grade } \\
(\mathrm{cm})\end{array}$} & \multicolumn{3}{|c|}{$\begin{array}{c}\text { Number of } \\
\text { diameter grade }\end{array}$} & \multicolumn{3}{|c|}{$\begin{array}{c}\text { Model-2 } \\
\text { prediction }\left(\mathrm{m}^{3}\right)\end{array}$} & \multicolumn{2}{|c|}{$\begin{array}{l}\text { Volume change } \\
2018 \text { to } 2014\left(\mathrm{~m}^{3}\right)\end{array}$} & \multicolumn{2}{|c|}{$\begin{array}{l}\text { Volume change } \\
2019 \text { to } 2018\left(\mathrm{~m}^{3}\right)\end{array}$} \\
\hline & 2014 & 2018 & 2019 & 2014 & 2018 & 2019 & Model -2 & $\begin{array}{c}\text { TLS } \\
\text { measurement }\end{array}$ & Model-2 & $\begin{array}{c}\text { TLS } \\
\text { measurement }\end{array}$ \\
\hline 10 & 2 & 0 & 0 & 0.32 & 0.00 & 0.00 & -0.32 & -0.29 & 0.00 & 0.00 \\
\hline 15 & 10 & 9 & 8 & 2.76 & 2.06 & 1.92 & -0.70 & -0.44 & -0.11 & -0.12 \\
\hline 20 & 13 & 9 & 8 & 7.36 & 5.81 & 5.35 & -1.55 & -2.41 & -0.46 & -0.78 \\
\hline 25 & 19 & 15 & 14 & 15.28 & 13.59 & 12.78 & -1.69 & -2.03 & -0.81 & -0.49 \\
\hline 30 & 13 & 18 & 15 & 15.34 & 21.47 & 22.67 & 6.13 & 6.97 & 1.20 & 1.53 \\
\hline 35 & 2 & 3 & 8 & 3.61 & 6.34 & 8.11 & 2.73 & 2.88 & 1.77 & 1.30 \\
\hline 40 & 1 & 0 & 1 & 1.14 & 0.00 & 1.60 & -1.14 & -1.75 & 1.60 & 1.65 \\
\hline 45 & 0 & 1 & 1 & 0.00 & 1.40 & 1.73 & 1.40 & 1.96 & 0.33 & 0.31 \\
\hline
\end{tabular}

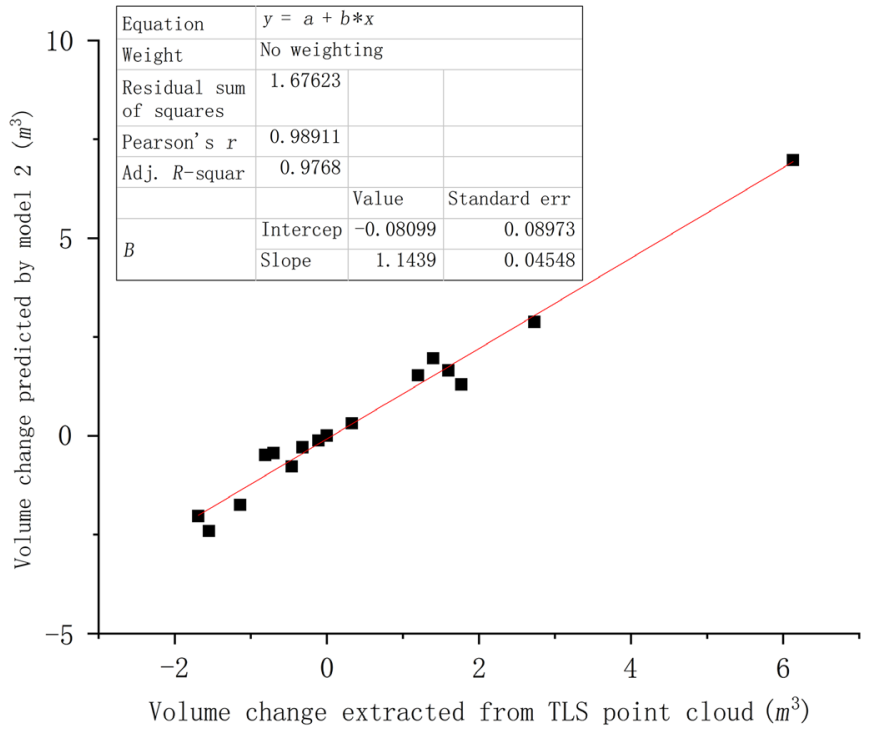

Fig. 7 Linear relationship between the modeled volume and the point cloud-extracted volume.

The scatter plot in Fig. 7 shows the variations in the modeled and TLS-extracted volumes. The linear relationship of the volume changes in the $\mathrm{DBH}$ grades between the modeled values and the values extracted from the point cloud was established and determined to have an intercept of -0.081 , a slope of 1.14 , and an $R^{2}$ of 0.98 (as shown in Fig. 7).

\subsection{Dynamic Analysis of the Tree Structure from Features}

\subsubsection{Individual tree changes and the growth rate}

Table 6 displays the individual changes in and growth rates of the tree height, $\mathrm{DBH}$, and tree volume in the three phases. Since the investigated periods spanned 4 years and 1 year, the growth rates are distinguished as 4-years and 1-year rates. The average rates of change among the three phases are as follows: tree height average growth rate of 0.36 to $0.39 \mathrm{~m}$; DBH average growth rate of 0.34 to $0.38 \mathrm{~cm}$; and volume average growth rate of 0.02 to $0.03 \mathrm{~m}^{3}$. Among these rates, 
Sun et al.: Multi-station LiDAR scanning-based hierarchical features for generation...

Table 6 Growth rates of the features for individual trees in the three periods.

\begin{tabular}{|c|c|c|c|c|c|c|}
\hline & \multicolumn{2}{|c|}{2014 to 2018} & \multicolumn{2}{|c|}{2018 to 2019} & \multicolumn{2}{|c|}{2014 to 2019} \\
\hline & $\begin{array}{l}\text { Growth } \\
\text { rate in } \\
4 \text { years }\end{array}$ & $\begin{array}{l}\text { Average } \\
\text { growth } \\
\text { rate }\end{array}$ & $\begin{array}{l}\text { Growth } \\
\text { rate in } \\
1 \text { year }\end{array}$ & $\begin{array}{l}\text { Average } \\
\text { growth } \\
\text { rate }\end{array}$ & $\begin{array}{l}\text { Growth } \\
\text { rate in } \\
5 \text { years }\end{array}$ & $\begin{array}{c}\text { Average } \\
\text { growth } \\
\text { rate }\end{array}$ \\
\hline Tree height (m) & 1.43 & 0.36 & 0.39 & 0.39 & 1.82 & 0.36 \\
\hline $\mathrm{DBH}(\mathrm{cm})$ & 1.57 & 0.39 & 0.34 & 0.34 & 1.91 & 0.38 \\
\hline Volume $\left(\mathrm{m}^{3}\right)$ & 0.08 & 0.02 & 0.03 & 0.03 & 0.11 & 0.02 \\
\hline
\end{tabular}

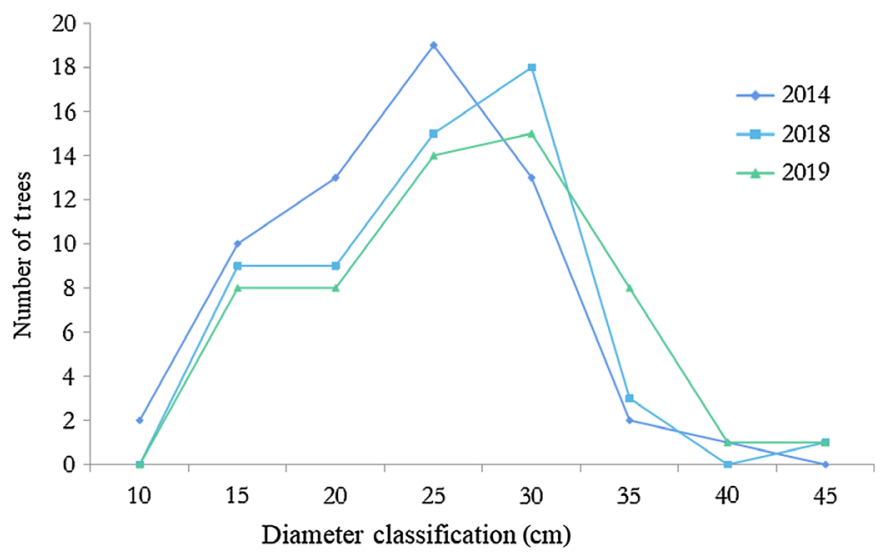

Fig. 8 Diameter grade distribution curves for 2014, 2018, and 2019.

the average growth rate of the tree height is greater than the average growth rate in the 4-year period of 2014 to 2018 . Moreover, the volume growth rates in all three periods are relatively average.

The variations in the diameter grades of the sample plot during the three periods are shown in Fig. 8. The diameter step was $5 \mathrm{~cm}$, and the initial diameter class was set to $5 \mathrm{~cm}$. As the number of trees was counted by diameter class, the peak of the diameter distribution curve shifted from $25 \mathrm{~cm}$ in 2014 to $30 \mathrm{~cm}$ in 2018 . Among the diameter classes, the shifted numbers of the 20 - and 25-cm diameter classes were relatively high.

\subsubsection{Height cumulative percentage analysis}

As shown in Fig. 9, the average $H_{z \%}$ of individual trees increases with the height in all three periods, with the first inflection point appearing at $25 \%$ (the height of the tree is $\sim 10 \mathrm{~m}$ ) with $H_{z \%}=10 \mathrm{~m}$. The second inflection point appears at 55\% (the height of the tree is $\sim 20 \mathrm{~m}$ ), with $H_{z \%}=20 \mathrm{~m}$. These results indicate that the hierarchical features can reflect the change in the stem shape, and these features appear regularly within all 5 years. The height cumulative percentages of $25 \%$ and $55 \%$ for the Chinese tulip (L. chinense) tree are the two inflection points for standing trees. As evidenced, the cumulative height percentage of the point cloud can reflect the change in the stem shape. The results of early studies in this study area showed that the stem shape of Liriodendron chinense varied greatly at different ages, which was mainly affected by the completeness of tree crown. The results show that 20 years is the optimal age for early selection of Liriodendron chinense. ${ }^{21}$ In order to improve trunk shape and increase stand volume per unit area, it is necessary to carry out timely monitoring.

A comparison with Fig. 6 shows the $H_{z \%}$ and stem shape variations, and the hierarchical feature clearly reflects the variation in the stem shape from the point cloud distribution with the tree height. Furthermore, $25 \%$ of $H_{z}$ is consistent with the periscope height. 


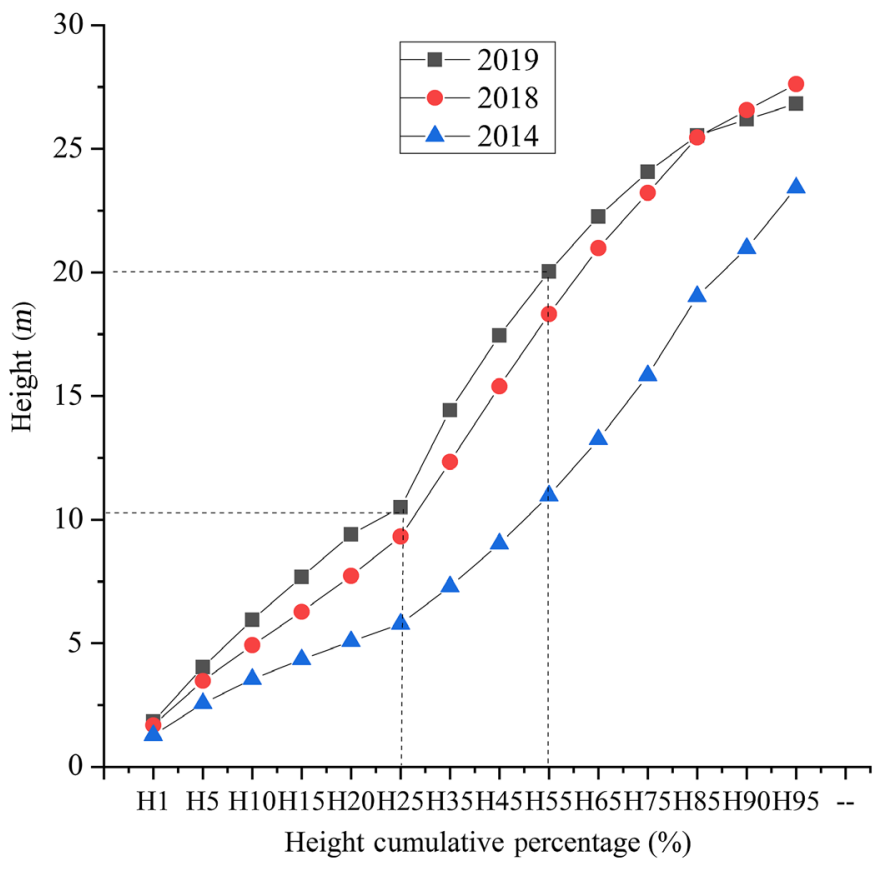

Fig. 9 Average $H_{z \%}$ for individual trees in three periods.

\section{Discussion}

As the use of TLS for dynamic monitoring of forest growth has increased, new challenges are arising in order to make the best use of the most detailed and accurate 3D forest data. ${ }^{22,23}$ From a hierarchical view of the point cloud of a standing tree, this research proposed the concept of the height cumulative percentage $\left(H_{z}\right)$ to study the association of $H_{z} \%$ with the stem volume. Through this parameter, structural variability can be quantified quickly and effectively, which then can provide the basis for forest planning and assessment of treatment impacts. $H_{z} \%$ was verified through stem analysis data as show in Fig. 6. The values of these parameters changed with the growth characteristics, the tree height, and upper diameter. Then we studied the potential of TLS by estimating the volume of $L$. chinense in the study area using point cloud hierarchical parameters. Table 3 lists the correlations of these characteristic parameters in the data from three periods and shows that among the parameters, $H_{50}$ and $H_{25}$ were mostly correlated with volume. Table 5 shows the volume models. The accuracies of models 14-2, 18-2, and 19-2 were improved by adding height cumulative percentage $\left(H_{z \%}\right)$ with the DBH factor (Table 4).

This research was inspired by the single-scan TLS case study ${ }^{23}$ but improved with a multiple-scan to obtain point cloud fully representing the tree trunk, thus to make it possible to study stem volume. As long as the $H_{Z}$ value is calculated, the change of the trunk shape can be reflected, and the next step of volume modeling can be carried out. Although the volume functions show less accurate than the DBH-H model but still acceptable compared with volume counted through point cloud with segment volume accumulations (Fig. 6), the estimation of volume variation also shows high accuracy (Fig. 7). Compared with other empirically based volume models, TLS derived allometric volume (with $R^{2}=0.94$ ). ${ }^{24}$ It confirmed that the changes in height-specific diameters and $\mathrm{h}$ are the most important factors when determining the increment in stem volume. ${ }^{7}$ Certainly, $H_{z \%}$ only describes the distribution of point cloud from the vertical level, whereas some old-growth stands have the highest horizontal structure variability. ${ }^{23}$ If there are parameters of the horizontal distribution structure, the volume model accuracy will be improved. This is also part of our on-going research, and new horizontal distribution indicators are being studied.

Repeated TLS data acquisition requires careful planning for ensuring point clouds with comparable quality. ${ }^{25}$ The type of sensor used to acquire data to study the growth of trees and forest stands has changed as technology advanced, and the comparison of data sets can be difficult due to differences in the sensor type used. ${ }^{15}$ This study attempts to solve the problem of multi-sensor 
data sources. The Leica equipment used in the first phase of the scan, with its high-precision settings and multi-site scanning with three bidirectional targets, was time-consuming. The latter two phases of scanning updated the equipment, eliminating the target, and reducing a lot of field work. However, the data processing was still very complicated, especially the extraction and calculation of the upper diameter of a single plant was time-consuming. The above-mentioned point cloud hierarchy parameters hopefully solve these problems. After registration, denoising, and normalization, the point cloud data of the three phases were uniformly distributed, and the order of magnitude of the point cloud was similar, with total points 19,211,863 in 2014, $19,774,289$ in 2018, and 19,509,890 in 2019. $H_{z}$ parameter reflects the hierarchical distribution of point clouds, and unit differences among multi-phase point clouds were removed by ratio calculation. At the same time, this parameter reflects the distribution of the upper diameter on the height of the tree, which is the integration of the three elements of volume. Whether this feature can reveal the trunk shape and whether $H_{z}$ is related to the planting density and species characteristics are worthy of further discussion. $H_{z}$ could also be discussed as a new parameter that can be obtained by laser scanning technology. We have tried to apply this parameter into different tree species, various stand management methods, and management densities and hope to investigate the parameter more specifically in the next paper.

Our research confirmed that TLS and explicit tree modeling show high potential for accurate stem volume estimation. ${ }^{26,27}$ Currently, airborne or unmanned aerial laser [airborne laser scan (ALS)] has the advantage of obtaining precise canopy point clouds, which has been confirmed in many studies. ${ }^{28,22}$ However, in forest resource monitoring, fusion of LiDAR data from terrestrial with different platforms and the use of fused point clouds has only been marginally explored. ${ }^{29}$ As Calders pointed out that co-registration of multi-source point clouds will be critical but can be achieved if enough common objects are presented to act as tie-points. ${ }^{30-33}$ It is a new idea to find the appropriate relationship from the vertical hierarchical distribution parameters of the treetrunk point cloud. Many studies have demonstrated that laser scanning data have an advantage in obtaining tree height ${ }^{27}$ and that ALS waveform can be converted into the corresponding canopy height contour; thus the height of each quantile can be calculated analogously in a sample canopy profile of the ground. ${ }^{32}$ Photogrammetry can also achieve the same data distribution. ${ }^{3}$ Therefore, whether vertical hierarchical parameters can be used as fusion features for multisource point cloud data are worthy of further discussion.

\section{Conclusion}

The structure of standing tree could be derived from TLS collected point cloud data, and a dynamic analysis could be performed through modeling with hierarchical features. The described research in this paper proposed a new parameter, the height cumulative percentage $\left(H_{z}\right)$, which is used to express the cumulative height of the first $\mathrm{m}$ points (from the bottom up to the first point) divided by all heights equal to $Z \% \mathrm{~m}$ points, with the first $\mathrm{m}$ point heights representing the $Z \%$ of $H$ of the point cloud. The $H_{z}$ value is closely related to the characteristic stumpage parameters and could be used to represent the dynamic forest structure. The results of this study contribute to the further development of TLS-based modeling and estimation methods for individual tree and forest growth, thereby improving the accuracy of forest inventories estimation and providing better tools for decision-making processes.

\section{Acknowledgments}

The authors would like to acknowledge the financial support by Co-Innovation Center for Sustainable Forestry in Southern China Nanjing Forestry University and the Priority Academic Programmer Development of Jiangsu Higher Education Institutions (PAPD). The author was grateful to Prof. Li Huogen the professor in Forest Faculty who offers great help in field test. And the author was also grateful to the anonymous referee for a careful checking of the details and for helpful comments that improved this paper. This study was funded by the Natural Science Foundation of Jiangsu Province (No. BK20191388), Jiangsu Provincial Postgraduate Research and Practice Innovation Program (No. KYCX20_0900), PAPD, and the China 
Postdoctoral Science Foundation (No. 2016M601822). The authors declare that they have no competing interests.

\section{References}

1. X. L. Liang et al., "International benchmarking of terrestrial laser scanning approaches for forest inventories," ISPRS J. Photogramm. Remote Sens. 144, 137-179 (2018).

2. T. Hilker, M. A. Wulder, and N. C. Coops, "Update of forest inventory data with lidar and high spatial resolution satellite imagery," Can. J. Remote Sens. 34, 5-12 (2014).

3. F. Pirotti and A. Vettore, "Vegetation filtering of waveform terrestrial laser scanner data for DTM production," Appl. Geomat. 5, 311-322 (2013).

4. N. Pfeifer, "Automatic reconstruction of single trees from terrestrial laser scanner data," ISPRS-Int. Arch. Photogramm. Remote Sens. Spatial Inf. Sci. 35, 114-119 (2004).

5. D. N. M. Donoghue et al., "Remote sensing of species mixtures in conifer plantations using LiDAR height and intensity data," Remote Sens. Environ. 110, 509-522 (2007).

6. X. L. Liang et al., "Terrestrial laser scanning in forest inventories," ISPRS J. Photogramm. Remote Sens. 115, 63-77 (2016).

7. V. Luoma et al., "Examining changes in stem taper and volume growth with two-date 3D point clouds," Forests 10(5), 382 (2019).

8. J. Muir et al., "An accuracy assessment of derived digital elevation models from terrestrial laser scanning in a sub-tropical forested environment," Remote Sens. 9, 843 (2017).

9. Y. Sun et al., "Deriving merchantable volume in poplar through a localized tapering function from non-destructive terrestrial laser scanning," Forests 7, 87-97 (2016).

10. R. Astrup et al., "Approaches for estimating stand-level volume using terrestrial lasers," Rev. Can. Recher. For. 44, 666-676 (2014).

11. V. Kankare et al., "Individual tree biomass estimation using terrestrial laser scanning," ISPRS J. Photogramm. Remote Sens. 75, 64-75 (2013).

12. S. M. K. Moorthy et al., "Terrestrial laser scanning for non-destructive estimates of liana stem biomass," For. Ecol. Manage. 456, 117751 (2020).

13. C. Mulverhill et al., "The utility of terrestrial photogrammetry for assessment of tree volume and taper in boreal mixedwood forests," Ann. For. Sci. 76(3), 83 (2019).

14. S. Kaasalainen et al., "Change detection of tree biomass with terrestrial laser scanning and quantitative structure modeling," Remote Sens. 6, 3906-3922 (2014).

15. T. Mengesha, M. Hawkins, and N. Nieuwenhuis, "Validation of terrestrial laser scanning data using conventional forest inventory methods," Eur. J. For. Res. 134(2), 211-222 (2015).

16. E. Che and M. J. Olsen, "Multi-scan segmentation of terrestrial laser scanning data based on normal variation analysis," ISPRS J. Photogramm. Remote Sens. 143, 233-248 (2018).

17. S. Srinivasan et al., "Multi-temporal terrestrial laser scanning for modeling tree biomass change," For. Ecol. Manage. 318, 304-317 (2014).

18. J. Wang et al., "Individual rubber tree segmentation based on ground-based LiDAR data and faster R-CNN of deep learning," Forests 10, 793 (2019).

19. X. Y. Meng, Forest Mensuration, China Forestry Publishing House, Beijing (2006).

20. L. Cao et al. "Estimating canopy structure and biomass in bamboo forests using airborne LiDAR data," ISPRS J. Photogramm. Remote Sens. 148, 114-129 (2019).

21. Z. Y. Hao et al., "Growth pattern and early selection of Liriodendron tulipifera," For. Res. 30(5), 878-885 (2017).

22. M. Mokroš et al., "Non-destructive monitoring of annual trunk increments by terrestrial structure from motion photogrammetry," PLoS One 15(3), e0230082 (2020).

23. D. Seidel, M. Ehbrecht, and K. Puettmann, "Assessing different components of threedimensional forest structure with single-scan terrestrial laser scanning: a case study," For. Ecol. Manage. 381, 196-208 (2016).

24. P. A. Klockow et al., "Allometry and structural volume change of standing dead southern pine trees using non-destructive terrestrial LiDAR," Remote Sens. Environ. 241, 111729 (2020). 
25. J. Liu et al., "Automated matching of multiple terrestrial laser scans for stem mapping without the use of artificial references," Int. J. Appl. Earth Obs. Geoinf. 56, 13-23 (2017).

26. Y. M. Li et al., "Retrieving the gap fraction, element clumping index, and leaf area index of individual trees using single-scan data from a terrestrial laser scanner," ISPRS J. Photogramm. Remote Sens. 130, 308-316 (2017).

27. C. H. Hu et al., "Trunk model establishment and parameter estimation for a single tree using multistation terrestrial laser scanning," IEEE Access 8, 102263-102277 (2020).

28. E. Næsset, "Predicting forest stand characteristics with airborne scanning laser using a practical two-stage procedure and field data," Remote Sens. Environ. 80, 88-99 (2002).

29. P. Wilkes et al., "Estimating urban above ground biomass with multi-scale LiDAR," Carbon Balance Manage. 13, 10 (2018).

30. X. Zhao et al., "Fusion of 3D LIDAR and camera data for object detection in autonomous vehicle applications," IEEE Sens. J. 20, 4901-4913 (2020).

31. C. A. Silva et al., "A principal component approach for predicting the stem volume in Eucalyptus plantations in Brazil using airborne lidar data," Forestry 89, 422-433 (2016).

32. K. Calders et al., "Terrestrial laser scanning in forest ecology: expanding the horizon," Remote Sens. Environ. 251, 112102 (2020).

33. K. Calders et al., "Implications of sensor configuration and topography on vertical plant profiles derived from terrestrial LiDAR," Agric. For. Meteorol. 194, 104-117 (2014).

Biographies of the authors are not available. 